\title{
Symmetry Principles in the Theory of Transport Properties with Special Reference to $p$ - Type Germanium
}

\author{
Lawætz, Peter
}

Published in:

PHYSICAL REVIEW

Link to article, DOI:

10.1103/PhysRev.166.763

Publication date:

1968

Document Version

Publisher's PDF, also known as Version of record

Link back to DTU Orbit

Citation (APA):

Lawætz, P. (1968). Symmetry Principles in the Theory of Transport Properties with Special Reference to $p$-Type Germanium. PHYSICAL REVIEW, 166(3), 763-769. https://doi.org/10.1103/PhysRev.166.763

\section{General rights}

Copyright and moral rights for the publications made accessible in the public portal are retained by the authors and/or other copyright owners and it is a condition of accessing publications that users recognise and abide by the legal requirements associated with these rights.

- Users may download and print one copy of any publication from the public portal for the purpose of private study or research.

- You may not further distribute the material or use it for any profit-making activity or commercial gain

- You may freely distribute the URL identifying the publication in the public portal

If you believe that this document breaches copyright please contact us providing details, and we will remove access to the work immediately and investigate your claim 


\title{
Symmetry Principles in the Theory of Transport Properties with Special Reference to $p$-Type Germanium
}

\author{
P. LAWAETZ \\ Physics Laboratory III, The Technical University of Denmark, Lyngby, Denmark
}

(Received 16 August 1967; revised manuscript received 6 October 1967)

\begin{abstract}
In order to solve the Boltzmann equation for low external electric and magnetic fields, taking into account details of band structure and scattering as in $p-\mathrm{Ge}$, the influence of crystal symmetry on scattering is discussed. The general symmetry properties of the scattering are considered and it is shown how this may facilitate the evaluation of the transport coefficients, especially those describing deviations from isotropy of the system. The method is applied to low-field magnetoconductivity and warm-carrier effects in cubic crystals like $p$-Ge.
\end{abstract}

\section{INTRODUCTION}

$T$ HE electrical transport theory of semiconductors with a band structure similar to that of $p$-type germanium has now reached a stage where the details of scattering mechanisms should be considered $^{1}$ for a satisfactory comparison of theory and experiment. The complexity of the band structure $e^{2,3}$ and its influence on the transition rates ${ }^{4,5}$ render an analytical treatment impossible, and numerical techniques must then be applied. ${ }^{6,7}$ In this case it has been found ${ }^{7}$ very useful, if not imperative, to use the maximum amount of information which can be gained from the symmetry of the crystal. The present paper is concerned with these symmetry principles and their application to the solution of the Boltzmann transport equation in some cases of interest.

\section{GENERAL PRINCIPLES}

We shall consider a system of independent charged particles in an infinite, homogeneous crystal. The oneparticle dispersion law $E=E(\mathbf{k})$ for the energy as a function of wave vector is assumed nondegenerate, but the results are easily extended to more complex band structures. The distribution function $f(\mathbf{k})$ is defined so that $f(\mathbf{k}) d \mathbf{k}$ denotes the number of carriers per unit (direct) volume in the (reciprocal) volume element $d \mathbf{k}$ at k. For the purpose of simplicity we assume a nondegenerate distribution. Then the rate of change in $f(\mathbf{k})$ due to scattering is

$\left(\frac{\partial f}{\partial t}\right)_{\mathbf{s c}}=\int d \mathbf{k}^{\prime}\left[P\left(\mathbf{k} \leftarrow \mathbf{k}^{\prime}\right) f\left(\mathbf{k}^{\prime}\right)-P\left(\mathbf{k}^{\prime} \leftarrow \mathbf{k}\right) f(\mathbf{k})\right]$,

${ }^{1}$ E. G. S. Paige, Progress in Semiconductors (Heywood and Co., Ltd., London, 1964), Vol. 8, p. 1.

${ }^{2}$ G. Dresselhaus, A. F. Kip, and C. Kittel, Phys. Rev. 98, 368 (1965).

${ }^{3}$ E. O. Kane, J. Phys. Chem. Solids 1, 82 (1956).

${ }^{4}$ G. L. Bir and G. E. Pikus, Fiz. Tverd. Tela 2, 2287 (1960) [English transl.: Soviet Phys.-Solid State 2, 2039 (1961)].

${ }^{5}$ G. L. Bir, E. Normantas, and G. E. Pikus, Fiz. Tverd. Tela 4, 1180 (1962) [English transl.: Soviet Phys.-Solid State 4, 867 (1962)].

${ }^{6}$ M. Tiersten, J. Phys. Chem. Solids 25, 1151 (1964).

${ }^{7}$ P. Lawaetz, thesis, The Technical University of Denmark, Lyngby, Denmark (unpublished). where $P\left(\mathbf{k}^{\prime} \leftarrow \mathbf{k}\right)$ is the rate of transitions from $\mathbf{k}$ to $d \mathbf{k}^{\prime}$ due to all scattering mechanisms (intercarrier scattering excepted, however).

\section{A. Symmetry Properties of Transition Rates}

We now assume that only the carriers are affected by external fields so that the scattering mechanisms are in thermal equilibrium with the crystal. Any phenomenon pertaining to the crystal in thermal equilibrium must have a symmetry compatible with that of the crystal. In the Brillouin zone this means the point group of symmetry operations of the crystal plus the timereversal operation the effect of which is equivalent to an inversion in $\mathbf{k}$ space. (For the present purpose we may disregard spin without loss of generality.) If the inversion is already present in the point group, no additional symmetry results from time reversal. In the following, this augmented point group is denoted by $R$. The symmetry of the scattering rates $P$ is expressed as $P$ being invariant under any operation $R$ in $R$ in the sense that

$$
P\left(R \mathbf{k}^{\prime} \leftarrow R \mathbf{k}\right)=P\left(\mathbf{k}^{\prime} \leftarrow \mathbf{k}\right) .
$$

Making use of this property, we find from Eq. (2.1)

$\partial$

$$
\begin{aligned}
& \frac{\partial}{\partial t} f(R \mathbf{k}) \\
& \quad=\int d \mathbf{k}^{\prime}\left[P\left(R \mathbf{k} \leftarrow \mathbf{k}^{\prime}\right) f\left(\mathbf{k}^{\prime}\right)-P\left(\mathbf{k}^{\prime} \leftarrow R \mathbf{k}\right) f(R \mathbf{k})\right] \\
& \quad=\int d \mathbf{k}^{\prime}\left[P\left(R \mathbf{k} \leftarrow R \mathbf{k}^{\prime}\right) f\left(R \mathbf{k}^{\prime}\right)-P\left(R \mathbf{k}^{\prime} \leftarrow R \mathbf{k}\right) f(R \mathbf{k})\right] \\
& \quad=\int d \mathbf{k}^{\prime}\left[P\left(\mathbf{k} \leftarrow \mathbf{k}^{\prime}\right) f\left(R \mathbf{k}^{\prime}\right)-P\left(\mathbf{k}^{\prime} \leftarrow \mathbf{k}\right) f(R \mathbf{k})\right] . \quad(2.3)
\end{aligned}
$$

This result is equivalent to the statement that the scattering operator $S_{\text {op }}$ defined by

$$
(\partial f / \partial t)_{\mathrm{sc}}=S_{\mathrm{op}} f(\mathbf{k})
$$

commutes with all operations $R$ in $R$, i.e., $S_{\text {op }}$ transforms according to the invariant irreducible representation $\Gamma_{i}$ of $R$. 
Owing to the completeness and orthogonality of the irreducible representations, we may write

$$
f(\mathbf{k})=\sum_{j \nu} f_{j v}(\mathbf{k})
$$

where $f_{j \nu}(\mathbf{k})$ belongs to the row $\nu$ in the irreducible representation $\Gamma_{j}$ of $R$. The summation is to be carried out over all possible $j$ and $\nu$.

Since $S_{\mathrm{op}} \sim \Gamma_{i}$, we observe that the scattering does not mix $f_{j v}(\mathbf{k})$ with different indices. This result has a number of important consequences for the solution of transport problems. We shall concentrate on the lowfield steady-state solution of the Boltzmann equation.

\section{B. Distribution Function for Low External Fields}

The steady-state transport equation governing the distribution function with electric field $\mathbf{F}$ and magnetic field $\mathbf{B}$ is, as usual,

where

$$
\left[\mathbf{F} \cdot \mathbf{F}_{\mathrm{op}}+\mathbf{B} \cdot \mathbf{B}_{\mathrm{op}}\right] f(\mathbf{k})=(\partial f / \partial t)_{\mathrm{sc}},
$$

$$
\begin{aligned}
& \mathbf{F}_{\mathrm{op}} \equiv(q / \hbar) \nabla_{\mathbf{k}}, \\
& \mathbf{B}_{\mathrm{op}} \equiv-(q / \hbar) \mathbf{V}(\mathbf{k}) \times \nabla_{\mathbf{k}}
\end{aligned}
$$

( $q$ being the charge of one particle), and

$$
\mathbf{V}(\mathbf{k})=(1 / \hbar) \nabla_{\mathbf{k}} E(\mathbf{k}) \text {. }
$$

In order to evaluate the low-field transport properties we expand the distribution function in a power series in the external fields $F$ and $B$,

$$
f(\mathbf{k})=\sum_{r s} \phi_{r s}(\mathbf{k})
$$

where $\phi_{00}=f^{0}(E)$ is the thermal-equilibrium distribution function, and, in general, $\phi_{r s} \propto F^{r} B^{s}$. Insertion of expression (2.10) in the transport equation (2.6) and separation of different powers in the fields give the following equations :

$$
\begin{gathered}
\phi_{00}=f^{0}(E), \\
\mathbf{F} \cdot \mathbf{F}_{\mathrm{op}} \phi_{r, 0}=S_{\mathrm{op}} \phi_{r+1,0}, \quad r \geqslant 0 \\
\mathbf{B} \cdot \mathbf{B}_{\mathrm{op}} \phi_{1, s}=S_{\mathrm{op}} \phi_{1, s+1}, \quad s \geqslant 0
\end{gathered}
$$

and for $r, s \geqslant 0$

$$
\begin{aligned}
\phi_{r+2, s+2} & =\phi_{r+2, s+2}(F)+\phi_{r+2, s+2}(B), \\
\mathbf{F} \cdot \mathbf{F}_{\mathrm{op}} \phi_{r+1, s+2} & =S_{\mathrm{op}} \phi_{r+2, s+2}(F), \\
\mathbf{B} \cdot \mathbf{B}_{\mathrm{op}} \phi_{r+2, s+1} & =S_{\mathrm{op}} \phi_{r+2, s+2}(B) .
\end{aligned}
$$

Note that the magnetic field does not influence the thermal-equilibrium distribution function which depends on energy only.

We observe from Eqs. (2.11)-(2.13) that the $\phi_{r s}$ may be determined, at least in principle, by a successive solution of these equations. A similar idea was developed by Schottky. ${ }^{8}$

\footnotetext{
${ }^{8} \mathrm{~W}$. Schottky, Festkörperprobleme (Vieweg, Braunschweig, 1962), Vol. 1, p. 316.
}

We now introduce the symmetry property of the scattering operator $S_{\text {op }}$ derived above. Since $S_{\text {op }}$ does not mix different irreducible representations (and rows therein) in $\phi$ expressed in a form like (2.5), the symmetry properties of the $\phi_{r s}$ are easily determined from the symmetry of $\mathbf{F}_{\mathrm{op}}$ and $\mathbf{B}_{\mathrm{op}}$. Thus, for the purpose of symmetry $S_{\mathrm{op}} \sim \Gamma_{i}$ may be taken equal to unity.

By inspection of (2.7) we find that $\mathbf{F}_{\text {op }}$ transforms according to the vector representation, while from (2.8) $\mathbf{B}_{\mathrm{op}}$ belongs to the pseudovector representation. These two representations are distinct since the group $\mathbb{R}$ always contains the inversion as a consequence of timereversal symmetry.

Because of the orthogonality properties of the irreducible representations and their rows and because $S_{\mathrm{op}} \sim \Gamma_{i}$, an equation of type (2.12) or (2.13), containing on the right-hand side a $\phi_{r s}$ of mixed symmetry (belonging to more than one irreducible representation), may be split up into equations with a $\phi_{r s}$ of pure symmetry in each. Thus the various symmetry parts of $\phi_{r s}$ are independent.

The advantage of the above method is that we may follow the different symmetry parts of $\phi_{r s}$ through the successive steps in the solution of the Eqs. (2.11)-(2.13). A primary consequence is that the solution procedure makes the choice of particular field configurations unnecessary, and secondly, in many low-field problems, only a limited number of representations will be generated by the field operators. Examples of this type are given in Sec. III. As shown in Sec. II C, this feature economizes the treatment of the scattering term in actual calculations.

Having found the possible symmetry properties of the distribution function (2.10), we can establish the connection between microscopic and macroscopic symmetry, the latter being expressed in the shape of the various conductivity tensors. The current density $\mathbf{j}$ is obtained from an integral of the type

$$
\mathbf{j}=\int d \mathbf{k} \mathbf{V} f(\mathbf{k}) .
$$

Since $\mathbf{V}$ as expressed in (2.9) belongs to the vector representation, only an $f_{j \nu}(\mathbf{k})$ of that representation contributes to the current as a consequence of the orthogonality of irreducible representations. On this basis, it is easy to construct the distinct transport coefficients corresponding to any function $\phi_{r s}$ of the expansion (2.10). We shall return to this in Sec. III in connection with cubic crystals.

\section{Further Consequences of the Symmetry}

In order to complete the picture of the effects of symmetry on the scattering, we shall return to the consideration of the transition rates. If these quantities are to be treated numerically as a consequence of their complexity, it is necessary to introduce a discretization 
in the continuous variables $\mathbf{k}$ and $\mathbf{k}^{\prime}$. Regarding the angular dependence, this purpose is conveniently achieved by expansion in a suitably chosen orthogonal system of harmonics. In order to make use of the symmetry properties described by the group $R$, we classify these harmonics according to the irreducible representations and rows therein. Let $Y_{j \nu}(n, \hat{k})$ be such a harmonic with $\hat{k}$ denoting the unit vector in the direction of $\mathbf{k}, j$ the irreducible representation, $\nu$ the row, and $n$ some order of harmonics within the same representation and row. The orthogonality and normalization are then expressed by

$$
\int d \hat{k} Y_{j v}(n, \hat{k}) Y_{j^{\prime} v^{\prime}}\left(n^{\prime}, \hat{k}\right)^{*}=4 \pi \delta_{n n^{\prime}} \delta_{j j^{\prime}} \delta_{\nu v^{\prime}},
$$

where the integration is performed over the unit sphere. The asterisk denotes complex conjugation. If $\mathbf{D}_{j}(R)$ is a unitary, irreducible representation of $R$, the classification of the harmonics as basis functions is given by

$$
Y_{j \nu}(n, R \hat{k})=\sum_{\mu} D_{j \nu \mu}(R) Y_{j \mu}(n, \hat{k}) .
$$

We now apply this harmonics expansion to the transition rates $P$, and so we write

$$
\begin{aligned}
P\left(\mathbf{k} \leftarrow \mathbf{k}^{\prime}\right)=\sum_{n n^{\prime} j^{\prime} \nu \nu^{\prime}} C_{j v, j^{\prime} \nu^{\prime}}\left(n, n^{\prime}\right) \\
\\
\quad \times Y_{j \nu}(n, \hat{k}) Y_{j^{\prime} \nu^{\prime}}\left(n^{\prime}, \hat{k}^{\prime}\right)^{*} .
\end{aligned}
$$

The coefficients $C$ may depend on $|\mathbf{k}|$ and $\left|\mathbf{k}^{\prime}\right|$. We then show that the symmetry property (2.2) limits the number of distinct coefficients $C$ in (2.17) considerably. In this treatment we neglect the suffixes $n$ and $n^{\prime}$ since the order has no connection with the symmetry. From the transformation properties (2.16) we find

$$
\begin{aligned}
P\left(R \mathbf{k} \leftarrow R \mathbf{k}^{\prime}\right)= & \sum_{j j^{\prime} \nu \nu^{\prime} \mu \mu^{\prime}} C_{j \mu, j^{\prime} \mu^{\prime}} D_{j \mu \nu}(R) \\
& \times D_{j^{\prime} \mu^{\prime} \nu^{\prime}}(R)^{*} Y_{j \nu}(\hat{k}) Y_{j^{\prime} \nu^{\prime}}\left(\hat{k}^{\prime}\right)^{*} .
\end{aligned}
$$

The left-hand sides of (2.17) and (2.18) are equal as a consequence of the invariance (2.2) of $P$, and because of the orthogonality of the harmonics we can identify the coefficients on the right-hand sides. Thus it follows from the symmetry that $C$ must satisfy

$$
C_{j \nu, j^{\prime} \nu^{\prime}}=\sum_{\mu \mu^{\prime}} C_{j \mu, j^{\prime} \mu^{\prime}} D_{j \mu \nu}(R) D_{j^{\prime} \mu^{\prime} \nu^{\prime}}(R)^{*} .
$$

By summing this equation over all $R$, we can make use of the great orthogonality theorem of group theory:

$$
\sum_{R} D_{j \mu \nu}(R) D_{j^{\prime} \mu^{\prime} \nu^{\prime}}(R)^{*}=h l_{j}^{-1} \delta_{j j^{\prime}} \delta_{\mu \mu^{\prime}} \delta_{\nu \nu^{\prime}},
$$

where $h$ is the order of the group and $l_{j}$ the dimension of the representation $\Gamma_{j}$. We then find from (2.19)

with

$$
C_{j \nu, j^{\prime} \nu^{\prime}}=A_{j} \delta_{j j^{\prime}} \delta_{\nu \nu^{\prime}}
$$

$$
A_{j} \equiv l_{j}^{-1} \sum_{\mu} C_{j \mu, j \mu} .
$$

The relation (2.21) is a necessary condition for the invariance (2.2) to hold. That it is also sufficient may be seen by insertion of (2.21) in (2.19). This gives the condition

$$
\sum_{\mu} D_{j \mu \nu}(R) D_{j \mu \nu^{\prime}}(R)^{*}=\delta_{\nu \nu^{\prime}}
$$

which just expresses the unitarity of the representation. Thus (2.21) is sufficient.

The relation (2.21) is readily interpreted: Different representations and rows are not mixed in the expansion (2.17), and coefficients corresponding to different rows within the same representation are equal. Thus

$$
P\left(\mathbf{k} \leftarrow \mathbf{k}^{\prime}\right)=\sum_{n n^{\prime} j \nu} C_{j}\left(n, n^{\prime}\right) Y_{j \nu}(n, \hat{k}) Y_{j \nu}\left(n^{\prime}, \hat{k}^{\prime}\right)^{*} .
$$

A comparison with the general expansion (2.17) then shows that a considerable reduction has taken place as a consequence of the symmetry requirements. However, for a complicated form of the transition rates the evaluation of the expansion coefficients $C_{j}$ may still be a formidable task. A further simplification is introduced by the fact that in many low-field problems we require knowledge of the $C_{j}$ 's belonging to only a limited number of representations $\Gamma_{j}$. This is shown below.

As we saw in Sec. II B, it is advantageous to split the Boltzmann equation of type (2.12) or (2.13) into equations with a $\phi_{r s}$ of pure symmetry appearing on the right-hand side. Let such a function be denoted by $f_{j v}(\mathbf{k})$ in order to conform with the expansion (2.5). By use of the orthogonality of irreducible representations, a consideration of the scattering term (2.1) combined with the expansion (2.23) then shows that:

(1) The $C$ coefficients contributing to the "out"scattering term $\int d \mathbf{k}^{\prime} P\left(\mathbf{k}^{\prime} \leftarrow \mathbf{k}\right) f_{j \nu}(\mathbf{k})$ belong to the invariant representation $\Gamma_{i}$.

(2) In the "in"-scattering term $\int d \mathbf{k}^{\prime} P\left(\mathbf{k} \leftarrow \mathbf{k}^{\prime}\right) f_{j \nu}\left(\mathbf{k}^{\prime}\right)$, the contributing $C$ should belong to the same representation as $f_{j \nu}\left(\mathbf{k}^{\prime}\right)$.

We conclude that in the solution of the equation for $f_{j \nu}(\mathbf{k})$ only the $C$ 's belonging to the $\Gamma_{j}$ and $\Gamma_{i}$ representations are needed. In a particular low-field problem it will usually be so that only a limited number of representations $\Gamma_{j}$ are excited by the field operators (as discussed in Sec. II B). Specific examples are given in Sec. III. We then require knowledge only about the $C$ coefficients belonging to these representations plus the invariant representation.

If for some special type of scattering additional symmetry occurs in the form of

$$
P\left(R \mathbf{k} \leftarrow \mathbf{k}^{\prime}\right)=P\left(\mathbf{k} \leftarrow \mathbf{k}^{\prime}\right),
$$


TABLE I. Irreducible representations of the full cubic group $\Gamma$.

\begin{tabular}{|c|c|c|c|}
\hline $\begin{array}{l}\text { Repre- } \\
\text { sentation }^{\mathrm{a}}\end{array}$ & Row $^{b}$ & Function ${ }^{\mathrm{c}}$ & Cubic harmonic ${ }^{d}$ \\
\hline \multirow{3}{*}{$\begin{array}{l}\Gamma_{1} \\
\Gamma_{2} \\
\Gamma_{12}\end{array}$} & & $\stackrel{\alpha}{\beta^{\prime}}$ & 1 \\
\hline & $s z$ & $\begin{array}{l}\beta^{\prime} \\
\gamma(s z)\end{array}$ & $\begin{array}{l}x^{4}\left(y^{2}-z^{2}\right)+\text { c.p. } \\
z^{2}-\frac{1}{3}\left(x^{2}+v^{2}\right)\end{array}$ \\
\hline & $a z$ & $\begin{array}{l}\gamma(s z) \\
\gamma(a z)\end{array}$ & $\begin{array}{l}z^{2}-\frac{1}{2}\left(x^{2}+y^{2}\right) \\
x^{2}-y^{2}\end{array}$ \\
\hline \multirow[t]{3}{*}{$\Gamma_{15}^{\prime}$} & $x$ & $\delta^{\prime}(x)$ & $y z\left(y^{2}-z^{2}\right)$ \\
\hline & $y$ & $\delta^{\prime}(y)$ & $z x\left(z^{2}-x^{2}\right)$ \\
\hline & $z$ & $\delta^{\prime}(z)$ & $x y\left(x^{2}-y^{2}\right)$ \\
\hline \multirow[t]{3}{*}{$\Gamma_{25}{ }^{\prime}$} & $x$ & $\epsilon(x)$ & $y z$ \\
\hline & $y$ & $\epsilon(y)$ & $z x$ \\
\hline & $z$ & $\epsilon(z)$ & $x y$ \\
\hline$\Gamma_{1}^{\prime}$ & & $\alpha^{\prime}$ & $x y z\left[x^{4}\left(y^{2}-z^{2}\right)+\right.$ c.p. $]$ \\
\hline \multirow{2}{*}{$\begin{array}{l}\Gamma_{2} \\
\Gamma_{12}\end{array}$} & & & $x y z$ \\
\hline & $\begin{array}{l}s z \\
a z\end{array}$ & $\begin{array}{l}\gamma^{\prime}(s z) \\
\gamma^{\prime}(a z)\end{array}$ & $\begin{array}{l}x y z\left[z^{2}-\frac{1}{2}\left(x^{2}+y^{2}\right)\right] \\
x y z\left(x^{2}-y^{2}\right)\end{array}$ \\
\hline \multirow[t]{3}{*}{$\Gamma_{15}$} & $x$ & $\delta(x)$ & $x$ \\
\hline & $y$ & $\delta(y)$ & $y$ \\
\hline & $z$ & $\delta(z)$ & $z$ \\
\hline \multirow[t]{3}{*}{$\Gamma_{25}$} & $x$ & $\epsilon^{\prime}(x)$ & $x\left(y^{2}-z^{2}\right)$ \\
\hline & $y$ & $\epsilon^{\prime}(y)$ & $y\left(z^{2}-x^{2}\right)$ \\
\hline & $z$ & $\epsilon^{\prime}(z)$ & $z\left(x^{2}-y^{2}\right)$ \\
\hline
\end{tabular}

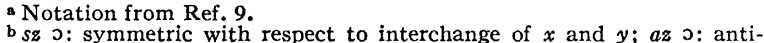
symmetric with respect to interchange of $x$ and $y$.

- Notation adapted from Ref. 10.

d Reference 10.

then group-theoretical arguments similar to those leading to Eq. (2.21) show that for $C_{j}$ belonging to $\Gamma_{j}$, either

or

$$
D_{j \nu \nu^{\prime}}(R)=\delta_{\nu \nu^{\prime}}
$$

$$
C_{j}=0 \text {. }
$$

It follows that $C_{j}$ is zero for all representations which are not invariant with respect to $R$. For all these representations, the "in"-scattering term vanishes and only the $\Gamma_{i}$ part of the $C$ coefficients is of interest. Usually, the operation $R$ in question is the inversion, and the symmetry property (2.24) is called momentum randomization. In this case all $C$ coefficients belonging to odd representations vanish.

\section{APPLICATION TO CUBIC CRYSTALS}

In the case of cubic crystals, the group $R$ is the full cubic group $\Gamma$. In the following, the 10 irreducible representations of $\Gamma$ are denoted according to Bouckaert et al., ${ }^{9}$ whereas functions belonging to particular irreducible representations follow the more compact notation of von der Lage and Bethe. ${ }^{10}$ The correspondence is shown in Table I which also explains the notation employed for rows.

Since the electric-field operator $\mathbf{F}_{\text {op }}$ in (2.7) transforms according to the vector representation, it follows that

$$
F_{\text {op }}{ }^{x} \sim \Gamma_{15 x} .
$$

Likewise for the magnetic-field operator (2.8),

$$
B_{\mathrm{op}}{ }^{x} \sim \Gamma_{15 x}{ }^{\prime} .
$$

${ }^{9}$ L. P. Bouckaert, R. Smoluchowski, and E. P. Wigner, Phys. Rev. 50, 58 (1936).

${ }^{10}$ F. C. von der Lage and H. Bethe, Phys. Rev. 71, 612 (1947).
Below, we shall use the principles developed in Sec. II B to study the symmetry properties of the distribution function in two important cases, the low-field magnetoconductivity and the warm-carrier problem.

\section{A. Low-Field Magnetoconductivity}

In cubic crystals the current density $\mathbf{j}$ may be expressed in the following way to first order in the electric field and to second order in the magnetic field:

$$
\begin{aligned}
\mathbf{j}=\sigma_{0}\left[\mathbf{F}+\alpha_{B}(\mathbf{F} \times \mathbf{B})+\beta_{B} \mathbf{F}\right. & B^{2} \\
& \left.+\gamma_{B} \mathbf{B}(\mathbf{F} \cdot \mathbf{B})+\delta_{B} \mathbf{T F}\right],
\end{aligned}
$$

where $\mathbf{T}$ is diagonal tensor with the elements $B_{x}{ }^{2}, B_{y}{ }^{2}$, and $B_{z}{ }^{2}$. This was shown in Ref. 11 by Seitz. We are going to show that the form (3.3) follows from the symmetry of the distribution function.

The effect of the field operators (3.1) and (3.2) on any distribution function of particular symmetry is given in the Appendix. From Eq. (2.12a) we find to first order in the electric field

$$
\begin{aligned}
& \phi_{10}=F_{x} \delta_{01}(x)+c . p . \\
& \delta_{01}(x) \leftrightarrow F_{\mathrm{op}} x f^{0}(E),
\end{aligned}
$$

and c.p. stands for cyclic permutations of $x, y$, and $z$. Here it has been used that $f^{0}$ has $\Gamma_{1}$ symmetry.

According to Eq. (2.12b), we now apply successive orders of the magnetic-field operator to $\phi_{10}$ and obtain in first order

$$
\begin{aligned}
& \phi_{11}=B_{x} F_{x}\left[\alpha_{11}{ }^{\prime}+\gamma_{12}{ }^{\prime}(a x)\right]+B_{x} F_{y}\left[-\delta_{13}(z)+\epsilon_{14}{ }^{\prime}(z)\right] \\
& +B_{x} F_{z}\left[\delta_{13}(y)+\epsilon_{14}^{\prime}(y)\right]+\text { c.p. , } \\
& \alpha_{11}{ }^{\prime}+\gamma_{12}^{\prime}(a x) \leftrightarrow B_{\mathrm{op}}^{x} \delta_{01}(x), \\
& \delta_{13}(x)+\epsilon_{14}{ }^{\prime}(x) \leftrightarrow B_{\mathrm{op}}^{z} \delta_{01}(y) .
\end{aligned}
$$

In second order of magnetic field we only retain the $\Gamma_{15}$ part, i.e., $\delta$ functions, because only these contribute to the current density and because we are not proceeding to higher orders.

$$
\begin{aligned}
& \phi_{12}\left(\Gamma_{15}\right)= B_{x}{ }^{2} F_{x}\left[\delta_{21}(x)+\delta_{22}(x)\right] \\
&+\left(B_{y}^{2}+B_{z}^{2}\right) F_{x}\left[-\delta_{23}(x)+\delta_{24}(x)\right] \\
&+\left(B_{x} B_{y} F_{y}+B_{x} B_{z} F_{z}\right)\left[\delta_{21}(x)-\frac{1}{2} \delta_{22}\right. \\
&\left.+\delta_{23}(x)+\delta_{24}(x)\right]+ \\
& \text { where } \delta_{21}(x) \leftrightarrow B_{\mathrm{op}}^{x} \alpha_{11}^{\prime}, \\
& \delta_{22}(x) \leftrightarrow B_{\mathrm{op}}^{x} \gamma_{12}^{\prime}(a x), \\
& \delta_{23}(x) \leftrightarrow B_{\mathrm{op}}^{z} \delta_{13}(y), \\
& \delta_{24}(x) \leftrightarrow B_{\mathrm{op}}^{z} \epsilon_{14}^{\prime}(y) .
\end{aligned}
$$$$
+\left(B_{x} B_{y} F_{y}+B_{x} B_{z} F_{z}\right)\left[\delta_{21}(x)-\frac{1}{2} \delta_{22}(x)\right.
$$$$
\left.+\delta_{23}(x)+\delta_{24}(x)\right]+ \text { c.p. , }
$$

Thus we find for the $x$ component of the current density, using (2.14) together with Eqs. (3.4), (3.6),

${ }^{11}$ F. Seitz, Phys. Rev. 79, 372 (1950). 
and (3.9),

$$
\begin{aligned}
j_{x} & =\sigma_{0}\left\{F_{x}+\alpha_{B}\left[F_{y} B_{z}-F_{z} B_{y}\right]+\beta_{B} F_{x}\left[B_{x}{ }^{2}+B_{y}{ }^{2}+B_{z}{ }^{2}\right]\right. \\
& \left.+\gamma_{B}\left[F_{x} B_{x}{ }^{2}+F_{y} B_{x} B_{y}+F_{z} B_{x} B_{z}\right]+\delta_{B} F_{x} B_{x}{ }^{2}\right\}
\end{aligned}
$$

with

$$
\begin{aligned}
& \sigma_{0}=\int d \mathbf{k} V_{x} \delta_{01}(x), \\
& \alpha_{B}=\sigma_{0}^{-1} \int d \mathbf{k} V_{x} \delta_{13}(x), \\
& \beta_{B}=\sigma_{0}^{-1} \int d \mathbf{k} V_{x}\left[-\delta_{23}(x)+\delta_{24}(x)\right], \\
& \gamma_{B}=\sigma_{0}^{-1} \int d \mathbf{k} V_{x}\left[\delta_{21}(x)-\frac{1}{2} \delta_{22}(x)\right. \\
& \delta_{B}=\sigma_{0}^{-1} \int d \mathbf{k} V_{x}\left[\frac{3}{2} \delta_{22}(x)-2 \delta_{24}(x)\right] .
\end{aligned}
$$

We observe that Eq. (3.14) is of the same form as the well-known expression (3.3). However, this result is rather trivial in itself. The important point is the relations (3.15)-(3.19) between the macroscopic conductivity parameters and the various symmetry components of the distribution function. This renders the consideration of particular field configurations unnecessary in actual calculations. In particular, the small longitudinal magnetoconductivity coefficients may be determined with improved accuracy as is evident from the following argument. These coefficients always involve $\beta_{B}$ and $\gamma_{B}$ in the combination $\beta_{B}+\gamma_{B}$, and we observe that such a combination does not contain a contribution from $\delta_{23}$. This function may be expected to be a predominant part of the distribution function of second order in magnetic field since the transverse effects are usually larger than the longitudinal. By the present analysis it should be possible to calculate the longitudinal and transverse coefficients with the same relative accuracy.

Concerning the practical solution of the transport equations involved in the magnetoconductivity calculation, the transition rates may be expanded in cubic harmonics ${ }^{10}$ in the way described in Sec. II C. Since the distribution function for this problem only contains symmetry parts corresponding to the odd representations $\Gamma_{1}{ }^{\prime}, \Gamma_{12}{ }^{\prime}, \Gamma_{15}$, and $\Gamma_{25}$, it follows from the discussion in Sec. II C that the expansion coefficients $C_{j}$ need only be calculated for these representations and the invariant representation $\Gamma_{\mathbf{1}}$. For the particular case of nonpolar optical-phonon scattering, the usual theory ${ }^{1,4}$ shows that this type of scattering is momentum randomizing. As shown in connection with Eq. (2.24), the harmonics expansion of the transition rates will then contain no odd representations, and so only the $\Gamma_{1}$ part is of interest in magnetoconductivity calculations.

\section{B. Warm-Carrier Conductivity}

In the warm-carrier problem we consider the lowestorder nonvanishing deviation from Ohm's law. The macroscopic symmetry of this phenomenon was investigated by Schmidt-Tiedemann ${ }^{12}$ and is easily derived from the magnetoconductivity relation (3.3) by replacement of $\mathbf{B}$ by $\mathbf{F}$,

$$
\mathbf{j}=\sigma_{0}\left[1+\left(\beta_{F}-\gamma_{F}\right) F^{2}+\gamma_{F} \mathbf{T}\right] \mathbf{F},
$$

where $\beta_{F}$ and $\gamma_{F}$ are the usual warm-carrier coefficients, ${ }^{12}$ and $\mathbf{T}$ is a diagonal tensor with the elements $F_{x}^{2}, F_{y}^{2}$, and $F_{z}^{2}$.

We now follow the procedure outlined in Sec. II and apply the electric-field operator (3.1). As in Eq. (3.4) we have the term of first order in the electric field

where

$$
\phi_{10}=F_{x} \delta_{11}(x)+\text { c.p. },
$$

$$
\delta_{11}(x) \leftrightarrow F_{\text {op }}^{x} f^{0}(E) .
$$

According to Eq. (2.12a) and the Appendix, the distribution function of second order in the electric field is

$$
\phi_{20}=F_{x}^{2}\left[\alpha_{21}+\gamma_{22}(s x)\right]+2 F_{y} F_{z} \epsilon_{24}(x)+\text { c.p. , }
$$

where

$$
\begin{aligned}
\alpha_{21}+\gamma_{22}(s x) & \leftrightarrow F_{\mathrm{op}}{ }^{x} \delta_{11}(x), \\
\delta_{23}{ }^{\prime}(x)+\epsilon_{24}(x) & \leftrightarrow F_{\mathrm{op}^{z}} \delta_{11}(x) .
\end{aligned}
$$

The $\delta^{\prime}$ functions cancel out in Eq. (3.23). In third order we find for the $\Gamma_{15}$ part (the only contribution to the current)

$$
\begin{aligned}
& \phi_{30}\left(\Gamma_{15}\right)=F^{2} F_{x} \delta_{31}(x)+\left(F_{x}^{2}-\frac{1}{2} F_{y}{ }^{2}-\frac{1}{2} F_{z}^{2}\right) F_{x} \delta_{32}(x) \\
& +2\left(F_{y}^{2}+F_{z}^{2}\right) F_{x} \delta_{34}(x)+c . p . \\
& \text { where } \\
& \qquad \begin{aligned}
\delta_{31}(x) \leftrightarrow F_{\mathrm{op}}{ }^{x} \alpha_{21}, \\
\delta_{32}(x) \leftrightarrow F_{\mathrm{op}}{ }^{x} \gamma_{22}(s x), \\
\delta_{34}(x) \leftrightarrow F_{\mathrm{op}}{ }^{z} \epsilon_{24}(y) .
\end{aligned}
\end{aligned}
$$

For the $x$ component of the current density we find using (2.14)

$$
\begin{aligned}
& j_{x}=\sigma_{0}\left[1+\left(\beta_{F}-\gamma_{F}\right) F^{2}+\gamma_{F} F_{x}^{2}\right] F_{x}, \\
& \sigma_{0}=\int d \mathbf{k} V_{x} \delta_{11}(x), \\
& \beta_{F}=\sigma_{0}^{-1} \int d \mathbf{k} V_{x}\left[\delta_{31}(x)+\delta_{32}(x)\right], \\
& \gamma_{F}=\sigma_{0}^{-1} \int d \mathbf{k} V_{x}\left[\frac{3}{2} \delta_{32}(x)-2 \delta_{34}(x)\right] .
\end{aligned}
$$

Again, the results of our method are in accordance with the macroscopic behavior (3.20). This agreement

${ }^{12}$ K. J. Schmidt-Tiedemann, Phys. Rev. 123, 1999 (1961). 
should only be regarded as a check. As in the discussion of magnetoconductivity in Sec. III A we have obtained a procedure of solution which does not rely on a specific field configuration. If the anisotropies connected with $\delta_{32}$ and $\delta_{34}$ are small, the present method shows that these may be isolated from the dominant $\delta_{31}$ part. It follows that in a numerical treatment these anisotropies may be determined with the same relative accuracy as the "isotropic" part $\beta_{F}$. In addition, we observe that the distribution function for this problem only contains symmetry parts corresponding to the representations $\Gamma_{1}, \Gamma_{12}, \Gamma_{25}{ }^{\prime}$, and $\Gamma_{15}$. According to the discussion of the harmonics expansion of the transition rates in Sec. II C, only expansion coefficients belonging to the above representations are needed in the actual calculation of $\beta_{F}$ and $\gamma_{F}$.

For the purpose of illustration let us take a look at a model which is commonly used as a simplified version of $p$-Ge. This is a simple band structure with isotropic acoustic- and optical-phonon scattering. As a consequence of the isotropy, $\gamma_{F}$ is zero, but this does not necessarily imply that the functions $\delta_{32}$ and $\delta_{34}$ in Eq. (3.33) vanish. In fact, a simple calculation shows that this is not the case. Thus it may not be a good approximation to neglect the $\delta_{32}$ term in (3.32) as is usually done in the so-called diffusion approximation where only zeroth- and first-order Legendre functions are retained. With isotropic scattering, only equations of $\Gamma_{1}$ type contain a nonzero "in"-scattering term (see Sec. II C). For pure acoustic scattering energy absorption is very inefficient so that the effect of $S_{\text {op }}$ on $\Gamma_{1}$ functions is small as compared to its effect on functions of a different symmetry. In this case $\delta_{31}$ (derived from $\alpha_{21}$ of the $\Gamma_{1}$ representation) is the predominant part of the distribution function in third order so that the diffusion approximation holds very well. Optical-phonon scattering, on the other hand, is a very efficient energyabsorption mechanism in $p$-Ge for carrier energies of the same order as the optical-phonon energy, so that $\boldsymbol{\delta}_{\mathbf{3 1}}$ is no longer large compared to $\delta_{32}$. Since this is the situation actually encountered in $p$-Ge, we conclude that the diffusion approximation is not sufficiently accurate for this material.

\section{CONCLUSION}

Although the method described in the preceding discussion is of general validity, it is not always the simplest path to follow in practice. The reason for this is connected with the explicit solution of the Boltzmann equation and may be illustrated by the case of the many-valley band structure. Here the usual and most convenient approach ${ }^{1}$ is a separate consideration of each valley. In our method, the valleys cannot be treated separately since this violates the cubic symmetry.

On the other hand, the present procedure is well suited for band extrema at $\mathbf{k}=0$. An important example of this type is the valence band edge in germanium where the complexity of the band structure and the scattering mechanisms has hitherto prohibited a detailed quantitative interpretation of hole-conduction phenomena. The present study provides us with a tool which makes possible a systematic approach to the solution of the Boltzmann equation, at least in the case of low fields.

In the numerical treatment of complex transport problems, some discretization of the continuous variables is always necessary. For the angular dependence we have seen in Sec. II C that an expression of the transition rates in harmonics transforming as basis functions of the irreducible representations of the point group $R$ will have a particular simple form if the scattering mechanisms are in thermal equilibrium. Since in most cases the scattering mechanisms are not directly influenced by the fields, only through the nonequilibrium distribution of the carriers, it follows that the above assumption is always fulfilled for sufficiently low external fields. If the coupling between the carriers and the scattering mechanisms is not too strong it may still be a good approximation to consider the latter in thermal equilibrium even in stronger fields. In this case the harmonics expansion in Sec. II C may be a profitable approach to the solution of transport problems with more general field strengths.

In a forthcoming publication we discuss actual calculations and results for the low-field magnetoconductivity properties of $p$-type germanium. These calculations will proceed according to the principles developed in the present work taking into account all relevant details of the band structure ${ }^{3}$ and the scattering. ${ }^{4}$

\section{ACKNOWLEDGMENTS}

The author is indebted to Professor N. I. Meyer for suggesting this work. Stimulating discussions with Dr. E. G. S. Paige are gratefully acknowledged.

\section{APPENDIX: SYMMETRY RULES FOR THE EFFECTS OF THE FIELD OPERATORS}

In Eq. (2.8) we defined the magnetic-field operator $\mathbf{B}_{\text {op }}$ with the symmetry (3.2),

$$
B_{\mathrm{op}}^{x} \sim \Gamma_{15 x^{\prime}} .
$$

We shall consider the effect of this operator when it operates on distribution functions of various symmetries corresponding to the irreducible representations (and rows therein) of the full cubic group $\Gamma$. We limit the discussion to odd representations because only these are of interest in connection with low-field magnetoconductivity. The following rules may be derived from group-theoretical considerations, but they may also be found from the basis functions listed in Table I, using the fact that

$$
B_{\mathrm{op}}^{x} \sim y z\left(y^{2}-z^{2}\right)
$$


The relations not shown below may be obtained by cyclic permutation of $x, y$, and $z$. Functions belonging to the same representation are formally identical if they have a common symmetry origin.

$$
\begin{array}{rlrl}
\Gamma_{1}^{\prime}: & B_{\mathrm{op}}^{x} \alpha^{\prime} & \leftrightarrow \delta(x) ; \\
\Gamma_{12 a}{ }^{\prime}: & B_{\mathrm{op}} \gamma^{\prime}(a x) & \leftrightarrow \delta(x), \\
B_{\mathrm{op}} \gamma^{\prime}(a x) & \leftrightarrow-\frac{1}{2} \delta(y)+\epsilon^{\prime}(y), \\
B_{\mathrm{op}}^{z} \gamma^{\prime}(a x) & \leftrightarrow-\frac{1}{2} \delta(z)-\epsilon^{\prime}(z) ; \\
B_{15}: & B_{\mathrm{op}} \delta(x) & \leftrightarrow \alpha^{\prime}+\gamma^{\prime}(a x), \\
B_{\mathrm{op}}{ }^{y} \delta(x) & \leftrightarrow \delta(z)+\epsilon^{\prime}(z), \\
B_{\mathrm{op}}{ }^{z} \delta(x) & \leftrightarrow-\delta(y)+\epsilon^{\prime}(y) ; \\
B_{\mathrm{op}} \epsilon^{\prime}(x) & \leftrightarrow \beta+\gamma^{\prime}(s x), \\
B_{\mathrm{op}}{ }^{y} \epsilon^{\prime}(x) & \leftrightarrow \delta(z)-\epsilon^{\prime}(z), \\
B_{\mathrm{op}} \epsilon^{\prime}(x) & \leftrightarrow \delta(y)+\epsilon^{\prime}(y) .
\end{array}
$$

$\Gamma_{2}^{\prime}$ and $\Gamma_{12 s}{ }^{\prime}$ are not used in the present work.
In the same way we derive the rules for the effects of the electric-field operator (2.7). Thus from (3.1) and Table I

$$
F_{\text {op }}{ }^{x} \sim \Gamma_{15 x} \sim x
$$

For the representations used in the present work we find the subsequent relations.

$$
\begin{aligned}
& \Gamma_{1}: \quad F_{\mathrm{op}}{ }^{x} \alpha \leftrightarrow \delta(x) ; \\
& \Gamma_{12 s}: \quad F_{\mathrm{op}}{ }^{x} \gamma(s x) \leftrightarrow \delta(x), \\
& F_{\text {op }}{ }^{y} \gamma(s x) \leftrightarrow-\frac{1}{2} \delta(y)+\epsilon^{\prime}(y), \\
& F_{\mathrm{op}}{ }^{z} \gamma(s x) \leftrightarrow-\frac{1}{2} \delta(z)-\epsilon^{\prime}(z) \text {; } \\
& \Gamma_{25}{ }^{\prime}: \quad F_{\text {op }}{ }^{x} \epsilon(x) \leftrightarrow \beta+\gamma^{\prime}(s x), \\
& F_{\mathrm{op}}{ }^{y} \epsilon(x) \leftrightarrow \delta(z)-\epsilon^{\prime}(z), \\
& F_{\mathrm{op}}{ }^{z} \epsilon(x) \leftrightarrow \delta(y)+\epsilon^{\prime}(y), \\
& \Gamma_{15}: \quad F_{\mathrm{op}}{ }^{x} \delta(x) \leftrightarrow \alpha+\gamma(s x), \\
& F_{\mathrm{op}}{ }^{y} \delta(x) \leftrightarrow \delta^{\prime}(z)+\epsilon(z), \\
& F_{\mathrm{op}}{ }^{z} \delta(x) \leftrightarrow-\delta^{\prime}(y)+\epsilon(y) \text {. }
\end{aligned}
$$

\title{
Contribution of Scattering of Polaritons by Phonons to Absorption of Light Waves in II-VI Crystals
}

\author{
W. C. TAIT AND R. L. WeIher \\ Central Research Laboratories, 3M Company, St. Paul, Minnesota
}

(Received 5 September 1967)

\begin{abstract}
Recent optical studies in II-VI compound single crystals have revealed discrepancies between actual absorption lines and the resonance frequencies obtained from a classical treatment of absorption and dispersion in insulators. This treatment neglects explicit dependence of the dielectric constant on the wave vector $\mathbf{k}$. In this paper, we calculate this wave-vector dependence of both the real and imaginary parts of the dielectric constant in a treatment which considers the scattering of the coupled exciton-photon (polarition) waves by phonons. Transmission, reflection, and absorption coefficients are then calculated for a typical II-VI crystal (CdS). The theoretical results obtained are found to be consistent with results given in the above optical studies. In particular, the intrinsic absorption lines are predicted to be wider and to peak at higher frequencies than generally expected from the classical treatment.
\end{abstract}

\section{INTRODUCTION}

$T$ HE absorption bands near the band edge have been extensively studied in the II-VI semiconductors. ${ }^{1}$ Some of these absorption bands are reported to be intrinsic in nature, being attributed to the formation of free excitons, which are mobile electronhole pairs bound together by their mutual energy of attraction. Also, absorption bands in the same range of wavelength have been reported which are attributed to the formation of excitons loosely bound on defect or foreign-ion complexes. These trapped or bound excitons for which crystal momentum is not a good quantum number are expected to behave like classical oscillators.

1 An excellent review of this work is given by D. C. Reynolds, C. W. Litton, and T. C. Collins, Phys. Status Solidi 9, 645 (1965); 12, 3 (1965)
Thus an accurate description of the absorption spectra associated with these bound excitons should be derivable from the classical treatment of absorption and dispersion in insulators. ${ }^{2}$

Noticeable discrepancies have been reported when this classical treatment has been extended to account for the absorption believed to be associated with the formation of free or intrinsic excitons. ${ }^{3}$ Recently, Park et al. ${ }^{4}$ noticed that a discrepancy of as much as $0.01 \mathrm{eV}$ existed between actual absorption lines in zinc oxide

${ }^{2}$ F. Seitz, Modern Theory of Solids (McGraw-Hill Book Co., Inc., New York, 1940), p. 633 .

${ }^{3}$ To our knowledge, the only paper in which the exciton absorption is calculated not using the classical method is that by A. A Demidenko and S. I. Pekar, Fiz. Tverd. Tela 6, 2771 (1964) [English transl.: Soviet Phys.-Solid State 6, 2204 (1965)].

${ }_{4}$ Y. S. Park, C. W. Litton, T. C. Collins, and D. C. Reynolds, Phys. Rev. 143, 143 (1966). 